\title{
A Taxonomy of Environmentally Scaffolded Affectivity
}

\author{
Sabrina Coninx \\ Research Training Group Situated Cognition, Institute for Philosophy II, \\ Ruhr University Bochum, Bochum, Germany \\ sabrina.coninx@rub.de \\ Achim Stephan \\ Institute of Cognitive Science, Osnabrück University, Osnabrück, Germany \\ achim.stephan@uos.de
}

\begin{abstract}
In this paper, we argue that the concept of environmental scaffolding can contribute to a better understanding of our affective life and the complex manners in which it is shaped by environmental entities. In particular, the concept of environmental scaffolding offers a more comprehensive and less controversial framework than the notions of embeddedness and extendedness. We contribute to the literature on situated affectivity by embracing and systematizing the diversity of affective scaffolding. In doing so, we introduce several distinctions that provide classifications of different types of environmentally scaffolded affectivity. Furthermore, we differentiate eight dimensions (e.g., trust, individualization, or intent) that allow us to evaluate the quality and effectivity of scaffolds in particular applications. On that basis, we develop a taxonomy using paradigmatic examples of affective scaffolding. This taxonomy enriches the current debate by emphasizing distinctions that are often conflated and by identifying fields of application that are commonly overlooked.
\end{abstract}

\section{Keywords}

affective scaffolding - situated affectivity - embeddedness - extendedness - userresource interaction - mind invasion - mind shaping 


\section{Introduction}

During the last decade, situated approaches to cognition ${ }^{1}$ have spread to our affective life and set out to study how our interaction with environmental entities contributes to affective phenomena. ${ }^{2}$ The concept of affective scaffolding is employed to characterize those cases in which we use or structure the environment in order to enable, support, enhance, or regulate specific affective experiences of ourselves or to shape the affective experiences of others. ${ }^{3}$ This paper contributes to the literature on situated affectivity in embracing and systematizing the diversity of environmental scaffolding. ${ }^{4}$ In particular, it enriches the current debate by emphasizing distinctions and fields of application that have not yet received sufficient attention.

The paper proceeds as follows. In section 2, we set out the traditional distinction between embeddedness and extendedness as it is typically discussed in the literature on situated affectivity and indicate some of the central problems related with this distinction. In section 3, we introduce the concept of affective scaffolding and lay out its usefulness for a better understanding of human affectivity. We outline three kinds of distinctions concerning the classification of affective scaffolding that are often conflated: (1) temporal scales that characterize different units of analysis in terms of relevant time lines

1 Cf. A. Newen, L. C. de Bruin, and S. Gallagher, “4E Cognition: Historical Roots, Key Concepts, and Central Issues," in Oxford Handbook of 4 E Cognition, ed. A. Newen, L. C. de Bruin, and S. Gallagher (Oxford: Oxford University Press, 2018), 1-15.

2 E.g., G. Colombetti and T. Roberts, "Extending the Extended Mind: The Case for Extended Affectivity," Philosophical Studies 172, no. 5 (2015): 1243-1263; P. E. Griffiths and A. Scarantino, "Emotions in the Wild: The Situated Perspective on Emotion," in The Cambridge Handbook of Situated Cognition, ed. P. Robbins and M. Aydede (Cambridge: Cambridge University Press, 2009), 437-453; J. Slaby, "Emotions and the Extended Mind," in Collective Emotions, ed. C. von Scheve and M. Salmela (Oxford: Oxford University Press, 2014), 32-46; A. Stephan, S. Walter, and W. Wilutzky, "Emotions beyond Brain and Body," Philosophical Psychology 27, no. 1 (2014): $65^{-81 .}$

3 E.g., G. Colombetti and J. Krueger, "Scaffoldings of the Affective Mind," Philosophical Psychology 28, no. 8 (2015): 1157-1176; J. A. Saarinen, "What Can the Concept of Affective Scaffolding Do for Us?," Philosophical Psychology 33, no. 6 (2020): 820-839; A. Stephan, "Critical Note: 3 E's are Sufficient, But Don't Forget the D," in The Oxford Handbook of 4 E Cognition, ed. A. Newen, L. C. de Bruin, and S. Gallagher (Oxford: Oxford University Press, 2018), 6o7-619; A. Stephan and S. Walter, "Situated Affectivity," in The Routledge Handbook of Phenomenology of Emotions, ed. T. Szanto and H. Landweer (Abingdon: Routledge, 2020), 299-311.

4 Some authors, such as Somogy Varga in Scaffolded Minds: Integration and Disintegration (Cambridge, MA: MIT Press, 2019), propose an understanding of scaffolding that does not merely apply to environmental entities, but also includes bodily and neural structures. We prefer to reserve the term 'scaffolding' for the relation between agent and environment. 
(e.g., environmental scaffolding of individuals or groups in microgenesis or sociogenesis); (2) reciprocity (uni-vs. bidirectional interaction) and direction of impact (user-resource interaction vs. mind shaping), which provide categorical classifications of different scaffolding relations; and (3) dimensions of affective scaffolding that indicate features (e.g., trust, individualization, or intent) on which the quality and the effectivity of the scaffolds might gradually rely. In section 4, we set out to develop a taxonomy of affective scaffolding by means of paradigmatic examples based on the previous three distinctions. Section 5 summarizes our conclusions.

Situated affectivity is primarily concerned with the question of how far an analysis in terms of the famous $4 \mathrm{E}$ 's - embodiment, enactment, embeddedness, and extendedness - can contribute to a better understanding of affective phenomena. In the last decade, the literature on situated affectivity found application to emotions, ${ }^{5}$ moods, ${ }^{6}$ temperaments, ${ }^{7}$ and affective disorders, ${ }^{8}$ as well as to less illuminated phenomena, such as pain. ${ }^{9}$ Situated approaches assume that affective phenomena are in a relevant sense codependent upon or co-constituted by entities beyond brain and body (e.g., objects, persons, structures, events, processes) and emphasize the relevance of the dynamic interaction between brain, body, and environment. Situated approaches are commonly contrasted

5 E.g., Griffiths and Scarantino, "Emotions in the Wild"; J. Krueger and T. Szanto, "Extended Emotions," Philosophy Compass 13, no. 4 (2016): 533-555; Slaby, "Emotions and the Extended Mind"; W. Wilutzky, S. Walter, and A. Stephan, "Situierte Affektivität," in Affektive Intentionalität, ed. J. Slaby, A. Stephan, H. Walter, and S. Walter (Paderborn: mentis, 2011), 283-320.

6 E.g., G. Colombetti, "The Embodied and Situated Nature of Moods," Philosophia 45, no. 4 (2017): 1437-1451.

7 E.g., Colombetti and Roberts, "Extending the Extended Mind."

8 E.g., S. N. Glackin, T. Roberts, and J. Krueger, "Out of Our Heads: Addiction and Psychiatric Externalism," Behavioural Brain Research 398 (2021): article 112936; J. Krueger, "Schizophrenia and the Scaffolded Self," Topoi 39 (2018): 597-6o9; J. Krueger, "Enactivism, Other Minds, and Mental Disorders," Synthese 198, supplement 1 (2021): 365-389; J. Krueger and G. Colombetti, "Affective Affordances and Psychopathology," Discipline Filosofiche 2, no. 18 (2018): 221-247; Varga, Scaffolded Minds.

9 E.g., S. Coninx and P. Stilwell, "Pain and the Field of Affordances: An Enactive Approach to Acute and Chronic Pain," Synthese (2021), https://doi.org/10.1007/s11229-021-03142-3; P. Stilwell and K. Harman, "An Enactive Approach to Pain: Beyond the Biopsychosocial Model," Phenomenology and the Cognitive Sciences 18, no. 4 (2019): 637-665. 
with approaches that characterize affective phenomena in purely cognitivist and internalist terms. ${ }^{10}$

The $4 \mathrm{E}$ 's are supposed to highlight different, though often complementary, aspects of the situatedness of affective phenomena. Just as there is no unitary class of situated approaches to cognition that can be identified with a set of commonly shared assumptions, there is no uniformity in the literature on situated affectivity. In this paper, we focus on the relationship between agent and environment as addressed in terms of embeddedness and extendedness. ${ }^{11}$ The distinction between the two notions concerns the question of whether affective phenomena are 'merely' codependent upon or causally coupled to extra-bodily entities (i.e., embedded), or whether they are 'really' co-constituted by or essentially based on such entities (i.e., extended). A situated approach to affectivity surely entails a commitment to embeddedness but not necessarily to extendedness. ${ }^{12}$

Several authors have applied a quite liberal understanding of extended affectivity based on the idea of 'tools for feeling' that allow us to steer our affective experiences into a certain direction (e.g., watching a movie to lift one's mood $)^{13}$ or self-stimulating loops of subject and environment interactions (e.g., the self-sustaining coupling of musician and instrument). ${ }^{14}$ However, authors with such a liberal understanding of extended affectivity seem to commit the notorious coupling-constitution fallacy, as they infer constitution from certain types of causal coupling. ${ }^{15}$ Liberals might respond that some forms of causal

10 Cf. Stephan and Walter, "Situated Affectivity," 300.

11 'Enactivism' denotes a diverse family of approaches that commonly highlight the interactive relation between an embodied agent and their environment; see, e.g., F. Varela, E. Thompson, and E. Rosch, The Embodied Mind: Cognitive Science and Human Experience (Cambridge, MA: MIT Press, 1991). Beyond this, the versions of enactivism differ from each other in various regards. See, e.g., G. Colombetti, "Enacting Affectivity," in The Oxford Handbook of $4 E$ Cognition, ed. A. Newen, L. C. de Bruin, and S. Gallagher (Oxford: Oxford University Press, 2018), 571-587. Embodied approaches are also quite diverse. Claims of embodiment may concern the functional reuse of brain areas, emphasize the phenomenal incorporation of one's body, or imply more or less demanding ontological commitments. See, e.g., Newen, de Bruin, and Gallagher, "4E Cognition," 5-6; Wilutzky, Walter, and Stephan, "Situierte Affektivität," 287-298. Cf. Griffiths and Scarantino, "Emotions in the Wild"; Stephan, Walter, and Wilutzky, "Emotions beyond Brain and Body," 78.

13 Slaby, "Emotions and the Extended Mind," 39.

14 Colombetti and Roberts, "Extending the Extended Mind," 1258-1259.

15 F. Adams and K. Aizawa, "The Bounds of Cognition," Philosophical Psychology 14 (2001): 4364; R. D. Rupert, Cognitive Systems and the Extended Mind (Oxford: Oxford University Press, 2009). 
coupling deserve to be considered 'constitutive,' for example, when there is a reliable, robust, and mutual influence between agent and environment that enables a novel form of steering or expression of affective phenomena inaccessible to the uncoupled individual. ${ }^{16}$ It remains to be asked, however, what we gain by considering these cases as forms of constitution rather than interesting cases of causal coupling. In general, liberal claims of affective extendedness are of little help as they lead to an inflation of constitution claims, or are misleading as they foster discussions at cross purposes. ${ }^{17}$

A general problem becomes obvious: even after years of dispute, the debate lacks a clear-cut and uncontroversial criterion that allows us to distinguish between cases of embeddedness and extendedness. In an attempt to tackle this problem, various authors have started to shift the discussion from the 'mark of the cognitive' (or the 'mark of the affective') toward the 'mark of constitution. ${ }^{18}$ Some argue that distinguishing between extendedness and embeddedness is at best a pragmatic issue ${ }^{19}$ that is irrelevant for our explanatory practice. ${ }^{20}$ In contrast, other researchers have tried to revive the debate by suggesting a concrete empirical criterion of what it is to be a constituent. ${ }^{21}$ An application of this criterion to cases of affective phenomena is yet to be implemented. Still others defend a fundamentally different conception of extendedness that refers to diachronic processes. ${ }^{22}$ As previously indicated, it remains an open question whether approaches that aim for a reconceptualizing of constitution can contribute to a better understanding of affective phenomena. The question of what makes something a constituent, and whether or how we can distinguish constitution from causation, are central questions of philosophy, irrespective of the debate surrounding embeddedness and extendedness. However, the paradigmatic cases discussed in the literature on situated affectivity can largely be studied independently of this distinction. Therefore, we argue for a shift in perspective away from the distinction

16 E.g., Colombetti and Roberts, "Extending the Extended Mind," 1258-1259; Krueger and Szanto, "Extended Emotions," 867-868.

17 Stephan and Walter, "Situated Affectivity," 304.

18 M. Baumgartner and W. Wilutzky, "Is It Possible to Experimentally Determine the Extension of Cognition?," Philosophical Psychology 30, no. 8 (2017): 1104-1125, here 1105, 1121.

19 Ibid., 1120-1122.

20 M. Sprevak, "Inference to the Hypothesis of Extended Cognition," Studies in History and Philosophy of Science 41, no. 4 (2010): 353-362.

21 B. Krickel, "Extended Cognition, the New Mechanists' Mutual Manipulability Criterion, and the Challenge of Trivial Extendedness," Mind and Language 35, no. 4 (2019): 539-561.

22 M. D. Kirchhoff, "Extended Cognition and the Causal-Constitutive Fallacy: In Search for a Diachronic and Dynamical Conception of Constitution," Philosophy and Phenomenological Research 40, no. 2 (2015): 320-36o. 
between extendedness and embeddedness toward an analysis in terms of scaffolding, which constitutes, in our view, an interesting subject of investigation in itself. The concept of scaffolding will be addressed in the next section.

\section{3}

\section{Affective Scaffolding}

The shift in perspective toward affective scaffolding allows us to highlight the relevance of explanations of affective phenomena that exceed brain and body, without accepting or rejecting a certain form of extendedness. Thus, the focus is on the question of whether we need to consider a certain environmental entity in order to provide a comprehensive account of the respective phenomenon, and not whether it is part of the physical realizer of this very phenomenon.

Scaffolding is the use or structuring of environmental entities (the scaffold) to enable, support, enhance, or regulate a certain activity, skill, or capacity (the scaffolded). ${ }^{23}$ Scaffolding relations may involve a heterogenous class of environmental entities that are causally related to the agent in various complex manners. ${ }^{24}$ In order to avoid trivial forms of scaffolding, the particular character of the scaffolded activity, skill, or capacity needs to depend, at least partly, on the character of the environmental scaffold. That is, the scaffold needs to be more than a mere background condition. ${ }^{25}$ This excludes from the list of scaffolds, for example, oxygen, which allows us to breathe, or light, which enables us to engage in visual perception. Further, the environmental entity needs to be more than a mere trigger. It needs to be used or structured by an agent, with or without explicit intention to do so. Consider, for example, a picture that triggers an emotional response. This only counts as a scaffold if a person hangs the picture in their apartment to enable such a reaction, or if it was placed there by others to enable it. It is not a scaffold if the person simply happens to come across the image.

23 A. Clark, Supersizing the Mind: Embodiment, Action, and Cognitive Extension (Oxford: Oxford University Press, 2008); K. Sterelny, "Minds: Extended or Scaffolded?, Phenomenology and the Cognitive Sciences 9 (2010): 465-481.

24 J. Sutton, C. B. Harris, P. G. Keil, and A. J. Barnier, "The Psychology of Memory, Extended Cognition, and Socially Distributed Remembering," Phenomenology and the Cognitive Sciences 9 (2010): 521-56o.

25 Varga, Scaffolded Minds, 50-52. 
Affective scaffolding is commonly distinguished from cognitive scaffolding, though they are interrelated in different manners. ${ }^{26}$ For present purposes, we may speak of affective scaffolding when aspects of the environment are used or structured to enable, support, enhance, or regulate the affective experiences of oneself or others. Affective scaffolding is not restricted to a particular (affective) domain, ${ }^{27}$ i.e., it might apply to emotions, moods, temperaments, affective disorders, and other affective phenomena. Furthermore, affective scaffolds are not restricted with respect to their structure, involving categories labeled as biochemical (e.g., food or drugs), representational (e.g., pictures or rituals), technical (e.g., computers or smartphones), sociobiological (e.g., other people or animals), sociotechnical (e.g., online platforms or social media), or socioinstitutional (e.g., conventions or norms). ${ }^{28}$

The concept of affective scaffolding is as such relatively flexible and may account for a variety of different cases concerning what is scaffolded and what scaffolds. Nonetheless, the literature previously focused primarily on a restricted number of examples, in particular concerning the short-term interaction of a single individual with an external resource. It is only recently that other examples have come more into focus, indicating the high number and diversity of cases we might account for in terms of affective scaffolding. ${ }^{29}$ This raises the question of whether such a broad characterization of affective scaffolding is in fact useful. For example, one might argue that it does not allow for a clear demarcation of scaffolded and nonscaffolded affectivity, and, thus, every affective phenomenon could turn out to be scaffolded in one way or another. ${ }^{30}$

Two remarks have to be made here. First, a more demanding characterization of affective scaffolding might run the risk of neglecting potentially

26 E.g., M. Maiese, "Affective Scaffolds, Expressive Arts, and Cognition," Frontiers in Psychology 7 (2016): article 359 .

27 Saarinen, "Concept of Affective Scaffolding," 823.

28 E.g., G. Colombetti, "Emoting the Situated Mind: A Taxonomy of Affective Material Scaffolds," The Journal for the Philosophy of Language, Mind and the Arts 1, no. 2 (2020): 215-236; Griffiths and Scarantino, "Emotions in the Wild"; Krueger, "Schizophrenia"; J. Krueger and L. Osler, "Engineering Affect: Emotion Regulation, the Internet, and the Techno-Social Niche," Philosophical Topics 47, no. 2 (2019): 205-231; F. León, T. Szanto, and D. Zahavi, "Emotional Sharing and the Extended Mind," Synthese 196 (2019): 4847-4867; Saarinen, "Concept of Affective Scaffolding"; J. Slaby, "Mind Invasion: Situated Affectivity and the Corporate Life Hack," Frontiers in Psychology 7 (2016): article 266; Sutton et al., "Psychology of Memory."

29 E.g., Glackin, Roberts, and Krueger, "Out of Our Heads"; Krueger and Osler, "Engineering Affect”; León, Szanto, and Zahavi, "Emotional Sharing”; Slaby, "Mind Invasion”; Varga, Scaffolded Minds.

Saarinen, "Concept of Affective Scaffolding," 830, 835. 
interesting phenomena and thus presuppose too narrow an understanding of affectivity and scaffolding. ${ }^{31}$ Second, the fact that our affective life is constantly scaffolded in complex ways is perhaps simply to be accepted. ${ }^{32}$ The crucial point, then, is to navigate the complexity of affective scaffoldings without trivializing scaffolding claims. Prominently, Jussi A. Saarinen suggests we should not problematize the broad and inclusive conceptualization of affective scaffolding, but instead define criteria for its adequate application:

I maintain that the concept of scaffolding is applied productively to affectivity when (a) it is clearly positioned vis-à-vis prior, apposite conceptualizations, (b) it reveals something new and significant about the way in which affective experience is supported by the environment, and (c) it enables us to expand on this revelation in a rigorous, comprehensive, and illuminating way. ${ }^{33}$

We consider this general approach to be very fruitful. One step on the path is to fully embrace the diversity of affective scaffolding and systematize paradigmatic cases in terms of a differentiated taxonomy. Such a taxonomy may contribute to a better understanding of why and when certain affective scaffolds are used or structured. ${ }^{34}$ Further, it may help us to exploit the variety of research areas for affective scaffolding while accepting that a useful application may strongly depend on both the particular research topics and interests. ${ }^{35} \mathrm{In}$ the remaining part of this section, we introduce three kinds of distinctions concerning the classification of different kinds of affective scaffolding that we consider relevant but not yet sufficiently elaborated. The respective criteria of distinction are introduced briefly here and will be discussed in more depth in Section 4 along with concrete examples.

First, affective scaffolding can unfold across different temporal scales. ${ }^{36}$ The relevant time lines may range from discrete short-term episodes of interaction

$31 \quad$ Saarinen, "Concept of Affective Scaffolding," 831-832; Slaby, "Mind Invasion," 4-8; Stephan and Walter, "Situated Affectivity," 305-308.

32 J. Sutton, "Scaffolding Memory: Themes, Taxonomies, Puzzles," in Contextualizing Human Memory: An Interdisciplinary Approach to Understanding How Individuals and Groups Remember the Past, ed. L. Bietti and C. Stone (Abingdon: Routledge, 2016), 187-205.

33 Saarinen, "Concept of Affective Scaffolding," 834.

34 See also Colombetti, "Emoting the Situated Mind."

35 See also Saarinen, "Concept of Affective Scaffolding," 835.

36 Griffiths and Scarantino, "Emotions in the Wild," 443-445; R. Menary, "Cognitive Integration: How Culture Transforms Us and Extends our Cognitive Capabilities," in The Oxford Handbook of 4 E Cognition, ed. A. Newen, L. C. de Bruin, and S. Gallagher (Oxford: Oxford University 
between agent and environment to developmental stages during a single lifetime, cross- and transgenerational social changes, and processes of cultural and biological evolution that may span hundreds or even thousands of years. Investigations of these different time scales naturally lean toward a focus on different agents in their interaction with the environment. Kinds of scaffolding may differ in terms of whether a single individual is involved or whether multiple individuals are collectively engaged. ${ }^{37}$ Individuals, small social groups (e.g., couples, peers, or families), complex social organizations (e.g., religious communities, companies, political movements, or nations), as well as whole populations can be considered as the agent who uses or structures the environment. ${ }^{38}$

Note that when we speak of families, companies, nations, or even populations as agents who use and structure the environment to influence human affectivity, we follow the explanatory practice, for example, in sociopolitical, historical, or legal contexts. By doing so, we do not claim that a family, company, nation, or population does act qua family, qua company, qua nation, or qua population. It is always particular individuals who are acting and whose affective phenomena are shaped. However, when their behaviors and affective responses are organized, structured, and coordinated in a certain way, it makes more sense to regard the entity that comprises the mutually interacting individuals as the agent - i.e., the relevant unit of analysis - than to consider the detailed behavior of a potentially large number of individual agents. Often, the latter does not present the relevant information in an easily digestible format and may be completely beyond what can be studied. ${ }^{39}$

Press, 2018), 187-215, here 197-202; Sterelny, "Minds," 476-479; Sutton, "Scaffolding Memory"; Varga, Scaffolded Minds, 49-76.

Colombetti and Krueger, "Scaffoldings," 1161; Krueger and Szanto, "Extended Emotions," 868870; Sterelny, "Minds," 477-479.

38 Slaby, "Mind Invasion," 7-9; Stephan and Walter, "Situated Affectivity," 308.

39 Many of the relevant cases to be outlined in the following might be located in a gray area of the common literature on (joint) agency, in that the interaction of the involved individual agents is not merely accidental but in some ways coordinated, although the individual agents might not explicitly aim to do so or be aware of them doing so (for a more detailed and nuanced discussion of the topic of implicit coordination, see J. Martens and L. Roelofs, "Implicit Coordination: Acting Quasi-Jointly on Implicit Shared Intentions," Journal of Social Ontology 4, no. 2 [2018]: 93-120). Moreover, the actions and affective phenomena of the involved individuals may relate to each other in multiple complex manners, being identical, complementary, or contrary. The central point that we want to make here is that in the investigation of strongly cooperating systems, it might prove useful to focus on the system level as the relevant unit of analysis. On that level, we can introduce so-called order parameters that can be seen as directing a process and that help to compress information. 
Taken together, our classification allows us to consider not only paradigmatic cases in which an individual makes use of the environment to influence their affective experiences in the here and now. It also allows us to include, for example, the evolutionary development of affective phenomena in the engagement of the human species with its environment, the generation, maintenance, and forwarding of narratives in social communities, or the acquisition and modification of the affective repertoire of an individual in childhood, adolescence, or adulthood. ${ }^{40}$ Affective scaffolding may be investigated on four temporal scales, labeled as microgenetic, ontogenetic, sociogenetic, and phylogenetic. ${ }^{41}$ Each scale focuses on a different unit of analysis. Which units are of relevance depends on our research interest, or more precisely, the research question we aim to answer with respect to affective scaffolding. An overview of the temporal scales, their paradigmatic units of analysis, and corresponding exemplary research questions is provided in Table 1.

Note that the temporal scales do not characterize where and when the scaffolded phenomenon and the environmental scaffold are physically realized. They indicate the time lines that we may consider in order to understand and explain the affective phenomena of the agent in focus. Furthermore, processes on different temporal scales relate to each other in complex ways. ${ }^{42}$ For example, ontogenetic processes are influenced by phylogenetic and sociogenetic factors, while ontogenetic developments are mediated by numerous microgenetic processes. ${ }^{43}$ At the same time, changes on different temporal scales show an asymmetry. Changes that are more global are always accompanied by changes at more local scales, whereas more local changes do not always add up to more global changes. ${ }^{44}$ For example, phylogenetic changes in the human species always involve changes on the sociogenetic and ontogenetic scales, but this relation rarely holds the other way around. Ontogenetic developments affect how we behave in certain situations; however, a single episodic engagement might not influence our further interactions.

See A. Stephan, "Varieties of Emergence in Artificial and Natural Systems," Zeitschrift für Naturforschung 53, no. 7-8 (1998): 639-656, here 650-652.

40 In a similar vein, Imke von Maur argues for the employment of synchronic-local as well as diachronic-global perspectives in situated approaches to affectivity in "Taking Situatedness Seriously. Embedding Affective Intentionality in Forms of Living" (Frontiers in Psychology 12 [2021]: article 599939). In the following, we are aiming for an even more detailed distinction. C. Sinha, "Language and Other Artifacts: Socio-Cultural Dynamics of Niche Construction," Frontiers in Psychology 6 (2015): article 1601.

42 Saarinen, "Concept of Affective Scaffolding," 833; Slaby, "Mind Invasion," 4.

43 Sinha, "Language and Other Artifacts."

44 For a similar idea, see S. de Haan, Enactive Psychiatry (Cambridge: Cambridge University Press, 2020). 
TABLE 1 Temporal scales: Units of analysis and exemplary research questions

\begin{tabular}{|c|c|c|c|c|}
\hline & Microgenesis & Ontogenesis & Sociogenesis & Phylogenesis \\
\hline $\begin{array}{l}\text { Units of } \\
\text { Analysis }\end{array}$ & $\begin{array}{l}\text { Episodic } \\
\text { interaction of } \\
\text { an individual } \\
\text { or a small } \\
\text { social group } \\
\text { with their local } \\
\text { environment }\end{array}$ & $\begin{array}{l}\text { Developmental } \\
\text { stages in the life } \\
\text { of an individual in } \\
\text { interaction with } \\
\text { the environment, } \\
\text { which may span } \\
\text { months, years, or } \\
\text { decades }\end{array}$ & $\begin{array}{l}\text { Interaction of } \\
\text { complex social } \\
\text { organizations with } \\
\text { their environment } \\
\text { that exceed the } \\
\text { lifespan of } \\
\text { particular } \\
\text { individuals }\end{array}$ & $\begin{array}{l}\text { Interactions } \\
\text { of populations } \\
\text { with their } \\
\text { environment } \\
\text { that usually } \\
\text { span numerous } \\
\text { generations }\end{array}$ \\
\hline $\begin{array}{l}\text { Exemplary } \\
\text { Research } \\
\text { Questions }\end{array}$ & $\begin{array}{l}\text { How does an } \\
\text { individual (or } \\
\text { couple) use the } \\
\text { environment } \\
\text { to change their } \\
\text { mood in the } \\
\text { here and now? }\end{array}$ & $\begin{array}{l}\text { How do children } \\
\text { acquire their } \\
\text { affective } \\
\text { repertoire? How } \\
\text { can individuals } \\
\text { learn to master } \\
\text { affective } \\
\text { disorders through } \\
\text { psychotherapy? }\end{array}$ & $\begin{array}{l}\text { What types of } \\
\text { affective involve- } \\
\text { ment do religious } \\
\text { practices support? } \\
\text { What affective } \\
\text { dynamics exist } \\
\text { between the } \\
\text { narratives of } \\
\text { nations with } \\
\text { long-standing } \\
\text { enmities? }\end{array}$ & $\begin{array}{l}\text { How has the } \\
\text { affective } \\
\text { repertoire } \\
\text { of humans } \\
\text { changed with } \\
\text { the emergence } \\
\text { of new social } \\
\text { structures, e.g., } \\
\text { in the process } \\
\text { of settling? }\end{array}$ \\
\hline
\end{tabular}

Second, we may consider affective scaffolds with respect to their reciprocity and direction of impact. These two categorical distinctions enable us to classify different kinds of affective scaffolding in terms of how and why certain entities are used or structured by the respective agent. Reciprocity indicates whether the relation between scaffold and scaffolded is best considered unidirectional or bidirectional. ${ }^{45}$ For example, watching a particular movie to lift one's mood is classified as unidirectional because the subject uses the movie to shape their affective experience without further reciprocal effect. In contrast, the intimate coupling between a mourning musician and their instrument is seen as bidirectional, because a feedback loop emerges between the affective experience of the musician and their affective expression in playing the instrument that in turn enables and deepens the affective experience and further guides their playing. ${ }^{46}$ The direction of impact allows us to distinguish between scaffolding

45 Saarinen, "Concept of Affective Scaffolding," 825; Stephan and Walter, "Situated Affectivity," 305-308.

46 Colombetti and Roberts, "Extending the Extended Mind," 1258-1259; Krueger and Szanto, "Extended Emotions," 867-868. 
as user-resource interaction, on the one hand, and as mind shaping, or even mind invasion, on the other. ${ }^{47}$ The agent who modifies the environment is not always identical to the agent whose affective life is scaffolded. In many paradigmatic cases, the relation of affective scaffolding is described as driven by an individual 'reaching outward' to use or structure the environment, ${ }^{48}$ as, for example, in the case of watching a movie to lift one's mood. However, an affective phenomenon might also originate in environmental entities when these are used by others to 'reach inward. ${ }^{49}$ This is the case, for example, when an individual is influenced by another person or group to undergo certain affective experiences or is 'brainwashed' to develop a certain affective repertoire. ${ }^{50}$

Third, scaffolding is a polyeidic concept; 51 i.e., it can be described in terms of numerous dimensions of gradual differentiation indicating the usefulness of the scaffold for the agent. ${ }^{52}$ Table 2 provides an overview of the dimensions of affective scaffolding. ${ }^{53}$ Different affective scaffolds instantiate different values on these dimensions, indicating different ways in which scaffolds can be

47 The term 'mind shaping' is prominently associated with Tadeusz Zawidzki, who argues that successful social interaction substantially relies on our capacity to mold the behavior of others, for example, by means of expert guidance or norm enforcement. See T. W. Zawidzki, "The Function of Folk Psychology: Mind Reading or Mind Shaping?, Philosophical Explorations 11, no. 3 (2008): 193-210. In the present paper, in the context of affective scaffolding, 'mind shaping' shall designate the exercise of external influence by others to steer the experience and behavior of a subject in a certain direction. Note, in addition, that we reserve the term 'mind invasion' for those cases in which the environmental scaffolding is contrary to the original goals and desires of an individual and prevents their personal development and flourishing (Slaby, "Mind Invasion," 2). The term 'mind shaping' is considered neutral in these regards.

48 E.g., Colombetti and Roberts, "Extending the Extended Mind," 1258-1259; Krueger and Szanto, "Extended Emotions," 866-868.

49 Stephan and Walter, "Situated Affectivity," 307-308.

50 There is a certain privilege to scaffolding. Some people have very little control over the spaces they inhabit (due to their personality, or due to socioeconomic factors and power relations) and, as such, might benefit less from scaffolding in terms of user-resource interactions. In contrast, they might be at greater risk from mind shaping and mind invasion (see also Krueger and Osler, "Engineering Affect," n. 5). We would like to thank an anonymous reviewer for stressing this point.

$51 \quad$ E. Borg, R. Harrison, J. Stazicker, and T. Salomons, "Is the Folk Concept of Pain Polyeidic?," Mind and Language 35, no. 1 (2020): 29-47.

$5^{2} \quad$ Sterelny, "Minds," 473-477.

53 Jussi A. Saarinen introduces a table titled "The multidimensionally scaffolded mind" (Saarinen, "Concept of Affective Scaffolding," 825). Our approach differs from his in the following three ways. First, we do not explicitly address differences in structure and domain. Both are of relevance for a comprehensive taxonomy of affective scaffolding but have already been widely discussed in the literature. Further, they might allow for categorical 


\section{Gradual differentiation}

(a) Trust

(b) Robustness

(c) Mineness

(d) Individualization

(e) Incorporation

(f) Awareness

(g) Intent

(h) Control
How reliable is the scaffold and our access to it? How stable is the integration of the scaffold into our affective life?

How deeply is the scaffold entrenched in our self-narrative?

How well is the scaffold adapted to our individual needs?

How phenomenally transparent or opaque is the scaffold?

To what extent are we aware of the scaffolding relation?

To what extent do we explicitly intend to use a scaffold for our purposes?

To what extent can we control the scaffolding relation?

more or less effective in enabling, supporting, enhancing, or regulating affective experiences. The following list does not claim to be complete and allows for overlaps and interrelations between the dimensions: (a) Trust concerns the reliability of a particular scaffold or a type of scaffold and the subject's access to it..$^{54}$ (b) Robustness characterizes the regularity with which a scaffold is engaged. ${ }^{55}$ (c) Mineness captures how closely the scaffold is integrated into one's self-narrative, i.e., how closely the scaffold is experienced with respect

classifications of different types of scaffolding rather than gradual differentiations along corresponding dimensions. Second, some of the dimensions addressed by Saarinen-i.e., temporality, individuality versus collectivity, and reciprocality—are not included in our table because they are considered as units of analysis on different temporal scales or addressed by the categorical distinctions of reciprocity and direction of impact. Third, the remaining dimensions - i.e., individualization, trust, and intent—are taken up, and further enriched, as indicated in Table 2. We argue for the usefulness of this more fine-grained distinction of the dimensions of affective scaffolding in section 4.2.

54 A. Clark and D. Chalmers, "The Extended Mind," Analysis 58 (1998): 10-23, here 17; Colombetti and Krueger, "Scaffoldings"; Sterelny, "Minds," 473-475.

55 Clark and Chalmers, "Extended Mind," 15; Colombetti and Roberts, "Extending the Extended Mind," 1245 . 
to who we perceive ourselves to be. ${ }^{56}$ (d) Individualization characterizes the degrees to which the particular scaffold is adapted to the individual agent. ${ }^{57}$ (e) Incorporation indicates to what extent the scaffold is phenomenally integrated into the affective experience. ${ }^{58}$ (f) Awareness indicates the degree to which people are consciously aware of the scaffolding relation. ${ }^{59}$ (g) Intent indicates whether the agent explicitly intends to use or structure an environmental resource to shape the affective experiences of themselves or others. ${ }^{60}$ (h) Control indicates the degree to which an agent can actively influence a scaffolding relation. ${ }^{61}$

On the basis of the previously indicated taxonomy, the concept of scaffolding remains as neutral as possible with regard to the choice of examples. At the same time, it also allows for a systematization of examples. In particular, it is important to maintain the distinction between temporal scales, reciprocity and direction of impact, and dimensions because (1) temporal scales allow for the characterization of different units of analysis depending on our research interest; (2) reciprocity and direction of impact allow for categorical classifications of different types of scaffolding relations on different temporal scales; and (3) dimensions indicate the different degrees to which scaffolding relations can be efficient. There is a danger that these differences will be lost if they are uniformly referred to as dimensions, as proposed, for example, by Colombetti and Krueger or Saarinen. ${ }^{62}$

A Taxonomy of Affective Scaffolding

In the following, we aim to provide examples of different kinds of affective scaffolding along the previously introduced distinctions. In principle, each case of scaffolding may be categorized with respect to the temporal scales and

56 M. Caravà and C. Scorolli, "When Affective Relation Weighs More Than the Mug Handle: Investigating Affective Affordances," Frontiers in Psychology 11 (2020): article 1928; R. Dings, "Understanding Phenomenological Differences in How Affordances Solicit Action," Phenomenology and the Cognitive Sciences 17 (2018): 681-699; R. Dings, "Meaningful Affordances," Synthese (2020), https://doi.org/10.1007/s11229-020-02864-o.

57 Colombetti and Krueger, "Scaffoldings," 1161; Sterelny, "Minds," 475-477.

58 Colombetti, "Enacting Affectivity," 583-584.

59 Saarinen, "Concept of Affective Scaffolding," 825, 827, 830; Stephan and Walter, "Situated Affectivity," 307.

6o Saarinen, "Concept of Affective Scaffolding," 825, 827, 832-834; Colombetti and Krueger, "Scaffoldings," 116o.

61 Saarinen, "Concept of Affective Scaffolding," 825, 83o, 833-834.

62 Colombetti and Krueger, "Scaffoldings"; Saarinen, "Concept of Affective Scaffolding." 
the corresponding units of analysis, the relation between scaffold and scaffolded in terms of reciprocity and direction of impact, and the degree to which they instantiate the features of trust, robustness, mineness, individualization, incorporation, awareness, intent, and control. We take the following approach.

In section 4.1, we first distinguish different kinds of affective scaffolding according to their direction of impact. Thus, we make a rough distinction between the categories of user-resource interaction and mind shaping. In a second step, we further classify these two categories in terms of their reciprocity as either uni- or bidirectional. We start with these two distinctions because we consider them most informative with respect to the question of how we can use environmental resources for our own purposes, but also how they can be used by others to shape our minds. In a third step, we fan out the respective categories in reference to the different temporal scales and pertinent corresponding research questions. We restrict the discussion to episodic engagements with the environment, ontogenetic developments, and sociocultural processes of a rather restricted scope in space and time. We do so to focus in sufficient detail on individuals in the context of their changing (modern) lifeworld. In section 4.2, we use some of the previously discussed examples to illustrate the differences between relevant dimensions and their contribution to scaffolding relations. We discuss the dimensions separately in order to guarantee a clear presentation in light of the diversity of different kinds of affective scaffolding.

\subsection{Direction of Impact, Reciprocity, and Temporal Scales}

In this subsection, we describe the different types of affective scaffolding in terms of their direction of impact and reciprocity and exemplify different fields of application along microgenetic, ontogenetic, and sociogenetic scales. We refer to paradigmatic examples while allowing for borderline cases that could be assigned to several categories.

\subsubsection{User-Resource Interaction}

We focus here on interactions between agent and environment that are best considered to originate within the agent and to stretch out from there into the environment through a process of resource usage. ${ }^{63}$ These user-resource interactions are further classified as either uni- or bidirectional.

63 Slaby, "Mind Invasion," 5-7; Stephan and Walter, "Situated Affectivity," 305-307. 


\subsubsection{Unidirectional User-Resource Interaction}

We consider kinds of affective scaffolding as unidirectional user-resource interactions when the agent is using or structuring an environmental entity in order to enable, support, enhance, or regulate their own affective experiences without thereby modifying and dynamically interacting with the scaffold, i.e., without initiating a causal feedback loop between the agent and the environmental resource, at least with respect to the unit of analysis that is in focus in the exemplary case. Further distinctions concern temporal scales of microgenesis, ontogenesis, and sociogenesis.

\subsection{Microgenetic Scale}

On the microgenetic scale, the relevant unit of analysis is the episodic interaction of an individual or small social group with their local environment. Many prominent examples in the literature fall into this category. ${ }^{64}$ We visit places that promise to steer our affective experience into an intended direction (e.g., visiting scenic places to ease our mood); we watch movies, listen to music, or read books that affect us in a certain way (e.g., reading The Lord of the Rings to experience the whole range of human emotions); and we put on certain clothes to increase our self-confidence (e.g., wearing a suit for a job interview to feel more confident). Similarly, we may think of a couple who jointly prepares dinner and lights candles to create a romantic atmosphere. ${ }^{65}$

\subsection{Ontogenetic Scale}

On the ontogenetic scale, one may consider those cases in which an agent uses or structures the environment to transform their general affective repertoire. ${ }^{66}$ For example, we may learn to regularly meditate or increase our awareness in order to improve our stress management abilities. Changes in our general affective repertoire are also often sustained by diachronic changes in our environment that promote transformative processes. ${ }^{67}$ For example, we may furnish our home to support affective experiences we aim for, or we may remove everything from our apartment that reminds us of our ex-partner to get over a breakup more easily. We may create particular places for mourning, such as shrines and mausoleums.

\footnotetext{
64 Colombetti and Krueger, "Scaffoldings"; Stephan and Walter, "Situated Affectivity," 305-306.

65 Although interactions between small social groups and environmental resources always also presuppose interactions between the people involved, we may consider this example as a form of unidirectional user-resource interaction as the couple is considered to be the relevant agent.

66 Stephan and Walter, "Situated Affectivity," 307.

67 Colombetti and Krueger, "Scaffoldings," 1159, 1169-1170, 1172.
} 


\subsection{Sociogenetic Scale}

On this scale, the relevant unit of analysis is a collective in its interaction with environmental resources that may exceed the life span of a single individual. It concerns the sociohistorical development of structures that enable the acquisition and expansion of the repertoire that members of a social group may use to shape their affective experiences. As a paradigmatic example, one may think of the generation and maintenance of religious or spiritual contexts, including material arrangements (e.g., building churches) and social conventions (e.g., rituals), which facilitate the experience of faith, compassion, or devotion. ${ }^{68}$ Further, in social communities, narratives may be jointly created and passed on that provide an affective resource for their members by enabling feelings of belonging or self-worth. Such narratives may include the origin myths of cities and nations (e.g., the tale of Romulus and Remus and the foundation of Rome), ethnic groups (e.g., the creation of humans out of trees described in the Edda), or religious communities (e.g., the discovery of the golden plates from which Joseph Smith claimed to have translated the Book of Mormon).

\subsubsection{Bidirectional User-Resource Interaction}

We consider user-resource interactions to be bidirectional when the agent uses or structures an environmental entity in order to enable, support, enhance, or regulate their own affective experiences while there exists a reciprocal coupling between scaffold and scaffolded. It is the particular nature of such a coupling that allows the agent involved to undergo the respective affective phenomena.

\subsection{Microgenetic Scale}

The relevant unit of analysis concerns an individual or group engaging in an episodic interaction with their local environment, enacting some kind of self-stimulating loop that has been set in place and is maintained in order to foster a certain emotional process. A repeatedly mentioned example of an episodic bidirectional user-resource interaction is a deeply mourning jazz musician whose "playing sets up a mutually constraining cycle of affective responding and expression: the qualities of the music performed, and of the actions and gestures initiated, feed back into the character of the musician's emotional experience, which in turn governs what he plays next." ${ }^{69}$ As another example, we may consider the use of video portals, such as YouTube, to episodically shape our mood. In response to our use, i.e., selecting, liking, and

68 Ibid., 1172.

69 Colombetti and Roberts, "Extending the Extended Mind," 1258. 
watching videos, the service suggests for our further consumption videos that are in line with our current preferences and habits (e.g., funny cat videos). This might again shape our ongoing use and the corresponding affective experiences that again influence our interaction with the device..$^{70}$

\subsection{Ontogenetic Scale}

The lifelong development and maintenance of bidirectional relationships with the environment plays an important role, especially as our relations within families, friendships, peers, and partnerships are built on the mutual shaping of our affective repertoire. In these cases, it is the existence and the particular nature of a coupling that allows the agent involved to enact some sort of affectivity along their life phases. For example, we may consider an old couple who have developed particular patterns of interaction that allow them to create cycles of mutual soothing and loving. ${ }^{71}$ This category of user-resource interaction also includes cases in which people get into cycles of behavior that reinforce negative emotions. This might apply to toxic relationships, in which the involved individuals are stuck in an ever-increasing dynamic of accusations, arguments, and anger. Self-reinforcing loops of affective scaffolding may also be associated with the generation and maintenance of psychopathologies ${ }^{72}$ or addictions, ${ }^{73}$ which lead to significant reductions in the affective repertoire of those concerned. An important part of treatment can be to break through these cycles and reestablish a person's ability to flexibly engage with their environment. ${ }^{74}$

\subsection{Sociogenetic Scale}

At this scale, the pertinent examples are cases in which the sociocultural narratives or practices of a particular group are developed and maintained in self-sustaining loops that are reinforced by the narratives and practices of other groups. We may think here of two groups that are hereditary enemies and each tell themselves stories about the perfidy of the other, perpetuating

70 Especially in the case of online platforms or social media, user-resource interaction and mind shaping usually seem to be two sides of the same coin, which we may consider from either one or the other perspective (see 4.1.2.2.1).

71 Stephan and Walter, "Situated Affectivity," 307.

72 S. de Haan, E. Rietveld, M. Stokhof, and D. Denys, "The Phenomenology of Deep Brain Stimulation-Induced Changes in OCD: An Enactive Affordance-Based Model," Frontiers in Human Neuroscience 7 (2013): article 653; Krueger and Colombetti, "Affective Affordances."

73 Glackin, Roberts, and Krueger, "Out of Our Heads."

74 Such processes can be facilitated by psychotherapeutic measures, which might also be considered as interesting cases of mind shaping (see 4.1.2.2.2). 
a mutual antagonism that fuels hostile narratives and practices on both sides. These relations may provide an emotional repertoire that cultivates feelings of belonging and pride as well as aggression and fear. ${ }^{75}$ Examples of this kind can be found many times in history, be it in the repeated armed conflicts between European states, the yet unresolved conflicts between parties in the Middle East, the formation of new political movements that define themselves against the 'establishment' and vice versa, or feuds between football clubs that are passed on. ${ }^{76}$ As history has shown, these bidirectional and mutually reinforcing relationships offer endless potential for escalation and can only be overcome with great effort (e.g., the development of Franco-German friendship).

\subsubsection{Mind Shaping}

In this section, we focus on interactions between agent and environment that are best considered to originate with structures in the environment and from there to 'reach inward' into the agent through a process of mind shaping, or even mind invasion. ${ }^{77}$ The following examples are further classified as either uni- or bidirectional. Please keep in mind that the distinction between examples of user-resource interactions and examples of mind shaping cannot always be sharply drawn and might partly depend on our scientific perspective.

\subsubsection{Unidirectional Mind Shaping}

We consider cases of affective scaffolding to exert unidirectional mind shaping when an agent is - intentionally or nonintentionally—scaffolding the affective experiences of another agent without there being a further mutual effect between the scaffolded and the scaffold, at least with respect to the unit of analysis that is the focus in the example case.

75 The examples presented here gain their presence and relevance on the level of groups and not with respect to the individual members that constitute them. What is important is that the groups gain a critical mass of enthusiasts who pass on the spirit or the memes of the group. This creates a certain form of dynamic that significantly shapes the affective life within and in interaction with a particular group. The dynamic is constituted by the behavior and affective responses of individuals, but best analyzed at the level of the larger social unit. It should be clear that the mutual interactions between the members of the group shape or invade in complex manners the affective life of both the group members and those who are considered outsiders. For example, individuals who resist such "streamlining" — such as the pacifists and true Europeans Stefan Zweig and Romain Rolland in the nationalist climate of World War I-often encounter harsh criticism and run the risk of social exclusion or marginalization.

76 See E. Halperin, Emotions in Conflict: Inhibitors and Facilitators in Peace Making (Abingdon: Routledge, 2016).

77 Slaby, "Mind Invasion," 7-11; Stephan and Walter, "Situated Affectivity," 307-308. 


\subsection{Microgenetic Scale}

There exist various cases in which agents, either individuals or groups, use the environment in order to episodically shape the affective experiences of others. For example, we may clean up the house and cook a favorite meal to cheer our partner up when they come home from work. We may put on a business suit for a job interview not only to make us feel more confident but also to evoke enthusiasm or admiration on the side of the potential employer. We may show up at a party with another person to make our ex-partner jealous. Interestingly, one could refer to almost exactly the same kind of examples as in unidirectional microgenetic user-resource interaction, except that the agent who uses or structures the environment is not identical with the agent whose affective experiences are enabled, supported, enhanced, or regulated..$^{78}$

\subsection{Ontogenetic Scale}

All human beings grow up in a certain material environment and are enculturated throughout their lives in particular social structures, including different family styles, group norms, religious regulations, or national laws. ${ }^{79}$ Humans are particularly competent mind shapers. ${ }^{80}$ Paradigmatic cases of mind invasion should be highlighted here in which an agent slowly 'hacks' another individual's mind so that they contribute to the agent's goal, although this may feel perfectly natural to the manipulated individual. ${ }^{81}$ As an extreme case, we may consider forms of radicalization. ${ }^{82}$ Radical organizations provide an environment that targets the inclinations and vulnerabilities of potential recruits in order to invade their minds and radicalize them in a transformative process. For example, through repeated lessons based on an extreme syllabus combining letters of the alphabet with affectively laden words and illustrations, ${ }^{83}$ children learn categorical thinking according to an us-versus-them mindset that facilitates feelings of anger, hatred, and disgust, but also of strength, pride, and

78 An example of episodic mind invasion would be the shaping of a political atmosphere at rallies, speeches, or meetings by means of the material arrangement (e.g., music, seating, billposting) or social setting (e.g., infiltration by apparent counterdemonstrators who act violently). For an insightful debate on political emotions and atmospheres and the complex manners in which they can be shaped, see L. Osler and T. Szanto, "Political Emotions and Political Atmospheres," in Shared Emotions and Atmospheres, ed. D. Trigg (Abingdon: Routledge, forthcoming).

79 Stephan and Walter, "Situated Affectivity," 307-308.

8o Zawidzki, "Function of Folk Psychology," 198-202.

81 Slaby, "Mind Invasion," 9.

82 H. Haq, S. Shaheed, and A. Stephan, "Radicalization through the Lens of Situated Affectivity," Frontiers in Psychology 11 (2020): article 205.

83 Haq, Shaheed, and Stephan, "Radicalization," 5. 
belongingness. As a second paradigmatic case, we may think of a company that creates a certain working atmosphere to increase employees' willingness to work. ${ }^{84}$ For example, the personnel management of a company might set up the working space in such a manner that there is no longer a clear separation of work and leisure (e.g., the Google Campus with bars, restaurants, and gyms), so that employees' conversations about work and their connection to their jobs exceed the official working hours.

\subsection{Sociogenetic Scale}

There are many occasions in which particular subgroups within a state (e.g., a political, economic, or religious subgroup) use or structure environmental resources, such as media or access to public resources, to shape the overall social climate or the affective repertoire of certain subgroups in their favor. ${ }^{85}$ The propaganda techniques that can be used for such purposes are manifold: illustrative distinctions between friend and foe, demonization of alleged enemies, idealization of certain forms of life (Lebensformen), distribution of misinformation, pseudo-scientific or pseudo-religious justification of one subgroup's superiority, creation of a cult of personality, etc. The goals of such forms of mind shaping might be to maintain a particular structure of power based on the presumed inequality of people of different origin, gender, skin color, religion, sexual orientation, or economic status. Such scaffolding may diametrically modulate the affective repertoire of the members of different social subgroups. For example, the agent may use structures, narratives, or rituals to enable self-confidence, faith, and feelings of belonging in the members of their own subgroup while reinforcing in others the internalization of their own worthlessness and inability, in order to increase their feelings of exclusion and hopelessness. While many of these cases point to deliberate mind shaping, we also encounter forms of mind shaping without explicit intent, ${ }^{86}$ as in cases of structural racism, sexism, or ableism that are culturally inherited and maintained.

\subsubsection{Bidirectional Mind Shaping}

The forms of mind shaping described previously always presuppose a certain form of receptivity on the side of the agent whose affective experiences are

84 Slaby, "Mind Invasion," 9.

85 At the sociogenetic scale, we refer to the general climate that prevails in an era or population and cannot be reduced to a single situation and its specific atmosphere (see n. 78). We take this distinction between 'climate' and 'atmosphere' from Osler and Szanto, "Political Emotions."

86 See also Martens and Roelofs, "Implicit Coordination." 
scaffolded. The agent must somehow 'allow' themselves to be mind-shaped or mind-invaded; one and the same strategy does not reach everyone, only those who are vulnerable or open to the offered scaffolds. However, although people may become aware of the processes that shape their mind and try to withdraw from them, the power seems to be rather with the agent who is using or structuring the environment. We speak of bidirectional mind shaping when the agent whose affective experiences are enabled, supported, enhanced, or regulated is more actively engaged in the processes of mind shaping and might even seek or contribute to it.

\subsection{Microgenetic Scale}

In football stadiums, groups of fans create an almost contagious atmosphere through drumming and chanting. Thus, when visiting a match, our affective experience might be strongly shaped by such behavior. For this very reason, we might expose ourselves to it, perhaps even contributing to the invasive character by singing along. A particularly interesting case may be the internet and social media. ${ }^{87}$ We voluntarily use platforms such as YouTube and Instagram to connect with people and accordingly regulate our affective experiences-a process that can be characterized as a case of user-resource interaction. Thereby, we might have the impression that in using such media we are shaping our emotions or moods only in accordance with our own principles. Often, however, we are not aware of how much other agents influence us at the same time-for example, through interface designs, algorithms, advertisements, or the strategic placement of posts and ads, which can render the use of social media also an instance of mind shaping. ${ }^{88}$ In turn, we ourselves continuously contribute to this system with our consumption and content, and by that may influence the affective experiences of other users.

\subsection{Ontogenetic Scale}

There are various forms of transformative processes in which we allow another agent to shape our minds and alter our general affective repertoire. Again, we might consider the internet and social media as illustrative examples. We integrate into our daily routines environmental resources that are designed, for example, to support addictive practices and enable patterns of dysfunctional emotion regulation. ${ }^{89}$ Often it is certain characteristics of the digital world that

87 Krueger and Osler, "Engineering Affect."

88 Ibid., $225^{-227 .}$

89 E.g. Glackin, Roberts, and Krueger, "Out of Our Heads"; Krueger and Osler, "Engineering Affect." 
promote more general changes in our affective repertoire, which, however, is not determined solely by such structures reaching inward. People actively use these resources, are shaped by characteristics of the platforms (e.g., anonymity, administration of comments, pop-up messages), ${ }^{90}$ and themselves become agents who shape the mind of others. ${ }^{91}$ Furthermore, many forms of psychotherapy fall into this category, as the concerned person actively seeks a place or person that 'reaches inward' in order to regain or enrich their own affective repertoire. ${ }^{92}$

\subsection{Sociogenetic Scale}

There are also cases in which one can speak of a bidirectional shaping regarding larger social groups and their extended cultural developments. In our examples of unidirectional influence, we have referred to propaganda techniques that can be used, for instance, by the government or a political group to create a certain social atmosphere or to maintain power structures. However, when it comes to those other members of society who benefit from these forms of scaffolding, it is not necessarily a one-way street. In some cases, parts of social groups seem to not only fall for certain kinds of propaganda, but actively expose themselves to their influence. This might apply to people who actively expose themselves to certain kinds of influence (e.g., by interacting on certain internet forums or participating in rallies) when, for example, they are playing with the idea of becoming members of a radical group or a political movement.

\subsection{Dimensions of Affective Scaffolding}

In this subsection, we elaborate on the dimensions that enable us to grade different types of affective scaffolding with respect to their quality and effectivity in concrete applications. As we shall see, these dimensions enable us to better understand how and when an agent relies on certain kinds of affective scaffolding. In particular, we aim for a more differentiated systematization of the dimensions of affective scaffolding than commonly proposed in the literature. For the sake of simplicity, we limit the following discussion to three classes of examples to show which roles the different dimensions play. Each dimension is addressed at least once, and it should become clear that certain dimensions might be more or less relevant to specific fields of application. Furthermore, we aim to emphasize that although the dimensions are interrelated, they should

9o Krueger and Osler, "Engineering Affect."

91 D. Valentini, A. M. Lorusso, and A. Stephan, "Onlife Extremism: Dynamic Integration of Digital and Physical Spaces in Radicalization," Frontiers in Psychology 11 (2020): article 524.

92 Stephan and Walter, "Situated Affectivity," 307. 
not be treated as identical. The selection of examples is rather pragmatic and clearly limited in order not to exceed the scope of the paper.

As a first category, we may consider examples of unidirectional userresource interactions on the microgenetic scale to address the dimensions of trust, robustness, and mineness. Some environmental entities are more trustworthy than others. For example, for some people, reading a book might be a more reliable source of emotional regulation than a visit to the theater and thus play a more central role in their affective life. In addition, some environmental entities are more frequently involved and thus fulfill a recurring, stable function. For example, meditation might be part of the daily relaxation routine of a person while hot baths are used for this purpose only on special occasions. Environmental factors that prove to be trustworthy are very likely to be those that we integrate as robust elements in our affective life. However, trustability and robustness are not identical. For example, chocolate might represent a more reliable affective resource than fruit, but in order to eat healthier, a person may have developed a sustainable routine that relies more strongly on fruit to lift their mood. As an additional feature, mineness denotes how close a scaffold is to who we believe ourselves to be. For example, we may listen to a song to lift our mood. A song that we associate with a positive memory of our adolescence might be better suited for this purpose than a song that has a catchy melody but has no personal significance. Note that trust and robustness do not necessarily imply mineness. Compare, for example, two subjects who regularly use pumpkin soup to comfort themselves after a stressful day. For one person, the pumpkin soup reliably enables the desired affective experience merely due to its biochemical properties (e.g., temperature and ingredients). For the other person, the soup reliably enables the desired affective experience because it reminds them of their beloved grandparents, even if this person might not even particularly like the taste of pumpkin soup. ${ }^{93}$ These cases might be equivalent in terms of trustability and robustness but differ in mineness.

As a second category, we may consider bidirectional user-resource interactions on the microgenetic and ontogenetic scale to address the dimensions of phenomenal incorporation and individualization. Concerning phenomenal incorporation, it may be said that in some cases affective scaffolds can become more or less transparent. For example, in the case of the musician, the instrument might no longer be experienced as an external tool but as the means through with they can achieve and express an affective experience.${ }^{94}$ Similarly,

93 Caravà and Scorolli, "When Affective Relation Weighs More," 3; Colombetti, "Emoting the Situated Mind," 231.

94 Colombetti, "Enacting Affectivity," 583. 
we might think of two grieving people at the funeral of a loved one embracing each other. ${ }^{95}$ Their boundaries may phenomenally disappear while becoming part of each other's experience of grief as well as comfort, and thus they reciprocally integrate each other and their affective experience. Concerning individualization, it seems that we often develop unique reciprocal dynamics with environmental entities. For example, our social relationships with significant others are often highly idiosyncratic. We develop unique dynamics in our interactions with parents, siblings, friends, partners, or colleagues that shape our affective repertoire. ${ }^{96}$ It is interesting that individualization and incorporation often go hand in hand but are not identical. For example, a musician may phenomenally incorporate an instrument that is not their own, and we might engage in idiosyncratic social relationships without the other being phenomenally transparent.

We would like to highlight that trust, robustness, mineness, phenomenal incorporation, and individualization are often mentioned in one breath. External resources that we reliably and regularly involve in our affective life are more likely to be adapted to our individual needs, become phenomenally transparent, and be experienced as close to who we are. Especially in these cases, the affective scaffold may be considered as deeply entrenched. ${ }^{97}$ In general, this seems to be associated with the possibility for smooth, spontaneous, and effortless interactions as well as feelings of familiarity. The ability to engage in these kinds of scaffolding relations is often disrupted in psychopathologies, such as depression, ${ }^{98}$ that are characterized by a loss in trustability with respect to interpersonal interactions and by an inability to incorporate and fluently interact with environmental resources, as well as feelings of isolation, alienation, meaninglessness, and impossibility. ${ }^{99}$

As a third category, we may consider mind shaping on the ontogenetic scale, considering both unidirectional and bidirectional examples to emphasize the characteristics of and differences between the dimensions of awareness,

95 Stephan, Walter, and Wilutzky, "Emotions beyond Brain and Body," 76.

96 Colombetti and Krueger, "Scaffoldings," 1171.

97 Cf. Sterelny, "Minds," 475-476, 480.

98 de Haan, Rietveld, Stokhof, and Denys, "Phenomenology"; R. E. Fabry, "Into the Dark Room: A Predictive Processing Account of Major Depressive Disorder," Phenomenology and the Cognitive Sciences 19 (2020): 685-704; Krueger and Colombetti, "Affective Affordances"; S. Varga and J. Krueger, "Background Emotions, Proximity and Distributed Emotion Regulation," Review of Philosophy and Psychology 4 (2013): 271-292.

99 K. Jacobs, A. Stephan, A. Paskaleva-Yankova, and W. Wilutzky, "Existential and Atmospheric Feelings in Depressive Comportment," Philosophy, Psychiatry, and Psychology 21, no. 2 (2015): 89-110. 
intent, and control. Since the agent whose affective repertoire is transformed is not identical to the agent who uses or structures the environment, both parties might come into focus in different manners. For example, in the case of a company providing a particularly structured workspace, members of the management team, but not the other employees, might be aware of such scaffolding. Furthermore, awareness does not imply that one has deliberately contributed to the described situation in the first place, as the involved parties might only become aware of it retrospectively. For example, one goal of family therapy can be to show parents how they have shaped, without explicit intent, the dysfunctional affective repertoire of their children and how they can overcome this. Further, awareness does not provide control for those whose minds are shaped or invaded. For example, we have some form of control over social media, in that we can use these platforms in a certain way and generate or consume certain content. However, when we become aware of the mechanisms of social media platforms, ${ }^{100}$ we find that the primary means of control is to avoid these platforms altogether.

As a final remark, we would like to highlight that an important step in personal and social development is to become aware of the different kinds of entities that we use to scaffold our affective life, and especially of those that are used by others to shape or even invade our minds. We are all part of complex social structures. As such, we contribute to the maintenance or modification of affectively relevant practices, rituals, or narratives and allow others to partly determine our affective experiences or transform our affective repertoire. This is not positive or negative per se, but awareness seems to be a first step toward gaining control as an individual or society and to consensually agree to such scaffolding relations. It must be the goal of further studies to show how awareness, intent, and control thereby relate to trust, robustness, mineness, incorporation, and individualization. For example, awareness and control seem particularly difficult when the relevant social structures are strongly linked to our identity and their modification is experienced as an attack on who we perceive ourselves to be.

We have argued for the usefulness of the concept of affective scaffolding and proposed a differentiated taxonomy in terms of temporal scales, reciprocity,

$100 \quad$ E.g., C. T. Nguyen, "Echo Chambers and Epistemic Bubbles," Episteme 17, no. 2 (2020): 141-161. 
direction of impact, and multiple dimensions. We believe that this taxonomy, together with preliminary work on the systematization of the domains and structures of affective scaffolding, ${ }^{101}$ can provide a better understanding of the many roles the environment can play in our affective life. In particular, we hope to draw attention to areas of research that so far have received little attention and from whose closer examination the debate could benefit, such as user-resource interaction and mind shaping on the sociogenetic scale. ${ }^{102}$ Further, we have outlined how different examples that are otherwise discussed independently from each other could be brought together in an overarching framework. This might lead to further fruitful discussions not only in philosophy but also in exchanges with the broader scientific audience and the public, for example, regarding the handling of political and religious radicalization, ${ }^{103}$ the possibilities and risks of the internet and social media, ${ }^{104}$ or the development of new therapeutic approaches. ${ }^{105}$

\section{Acknowledgments}

Special thanks to Regina Fabry and Sven Walter as well as Hina Haq, Gregor Hörzer, Louis Loock, Imke von Maur, Leonardo Massantini, Daniele Valentini, and other members of the Reading Club "Affectivity" of Osnabrück University for their feedback on earlier versions of the paper. We would also like to thank the anonymous reviewers for their helpful and thoughtful comments. Funded by the Deutsche Forschungsgemeinschaft (DFG, German Research Foundation) - project number GRK-2185/1 (DFG Research Training Group Situated Cognition).

\footnotetext{
101 Colombetti, "Emoting the Situated Mind"; Colombetti and Krueger, "Scaffoldings"; Colombetti and Roberts, "Extending the Extended Mind"; Saarinen, "Concept of Affective Scaffolding." First steps in this direction have recently been made: e.g., Osler and Szanto, "Political Emotions."

103 Haq, Shaheed, and Stephan, "Radicalization."

104 Krueger and Osler, "Engineering Affect"; Valentini, Lorusso, and Stephan, "Onlife Extremism."

105 Glackin, Roberts, and Krueger, "Out of Our Heads."
} 